\title{
INVESTIGACIONES
}

\section{Escritura académica de un ensayo mediado por el aprendizaje colaborativo virtual ${ }^{*}$}

\author{
Academic writing of an essay mediated by virtual collaborative learning \\ Escrita acadêmica de um ensaio mediado pela aprendizagem virtual colaborativa
}

\begin{abstract}
Beatriz Figueroa, Mariana Aillon. ${ }^{a}$
${ }^{a}$ Departamento de Currículum e Instrucción, Facultad de Educación, Universidad de Concepción, Chile. Fono: 41-2207235. Correos electrónicos: bfiguero@udec.cl, maillon@udec.c1
\end{abstract}

\begin{abstract}
El estudio expuesto a continuación da cuenta de una investigación realizada el primer semestre del año 2012, en un grupo de 19 estudiantes del Programa de Magíster en Educación de la Facultad de Educación de la Universidad de Concepción. El grupo cursó la asignatura "Leer y escribir en el siglo XXI”, que tiene como objetivo potenciar la escritura académica por medio de la producción de un ensayo científico. Gracias al mejoramiento de anteriores experiencias metodológicas, se ha elaborado un diseño didáctico que articula el desarrollo de la escritura académica con apoyo tecnológico, para lo cual se cuenta en la actualidad con un número de lecciones que van sistemáticamente haciendo progresar la escritura del texto con apoyo de un esquema digital de escritura (EDE).
\end{abstract}

Palabras clave: escritura académica, tecnología, diseño didáctico.

ABSTRACT

The study presented below is related to a research work carried out during the first semester of year 2012 concerning a group of 19 students belonging to the Education Master program of the Faculty of Education of Universidad de Concepción, Chile. The group took the course "To read and write in the XXI century", whose purpose is to enhance academic writing by means of the production of a scientific essay. Thanks to the improvement of previous methodological experiences a didactic outline has been designed; it links the development of the academic writing with the support of technological tools which includes a series of lessons that systematically help the students to advance in the writing work of the text with the backing of a digital writing scheme (DWS, or EDE, esquema digital de escritura in Spanish).

Key words: academic writing, technology, didactic design.

\section{RESUMO}

O estudo expõe uma investigação realizada no primeiro semestre do ano 2012, em um grupo de 19 estudantes do Programa de Mestrado em Educação da Faculdade de Educação da Universidade de Concepción. O grupo cursou a disciplina "Ler e escrever no século XXI" com o objetivo de potenciar a escrita acadêmica por meio da produção de um ensaio científico. Graças ao melhoramento das experiências metodológicas anteriores, elaborou-se um projeto didático que articula o desenvolvimento da escrita acadêmica com apoio tecnológico, para o qual, na atualidade, se conta com um número de lições que vão melhorando sistematicamente a escrita do texto com apoio de um esquema digital de escrita (EDE).

Palavras chave: escrita acadêmica, tecnologia, projeto didático.

Los resultados presentados en este artículo corresponden al Proyecto Fondecyt Regular № 1110909 “Alfabetización académica: el hipertexto una herramienta para mejorar los aprendizajes en la formación de profesores" (2011- 2013), financiado por la Comisión Nacional de Investigación Científica y Tecnológica (CONICYT) de Chile. 


\section{CONSIDERACIONES PREVIAS}

"Lo que se aprende es inseparable del modo en que se aprende" (Marton \& Booth, 1997).

La escritura ha sido utilizada durante mucho tiempo como proceso y producto del pensamiento crítico riguroso, dimensiones cognitivas que se potencian por la conectividad y flexibilidad de la interacción apoyada por los medios tecnológicos (Garrison y Anderson, 2005; Bereiter, 2002). A continuación describiremos los elementos que componen el diseño didáctico, donde se articulan la producción de un género discursivo, el ensayo, mediado por la tecnología y el aprendizaje colaborativo.

\subsection{PRODUCCIÓN DE UN ENSAYO}

En la experiencia, la producción de un ensayo como texto argumentativo requirió de lecturas críticas como base del conocimiento sobre el tema del escrito, seguido de un proceso de reflexión que permitió a cada sujeto elaborar su perspectiva personal del contenido a partir de la revisión bibliográfica especializada. Dicha reflexión, en una primera instancia, se tradujo en una pregunta de investigación, seguida de un mapa mental que, como ordenador gráfico, facilitó la visualización de la síntesis con las ideas más relevantes seleccionadas de las fuentes bibliográficas, para que, en una segunda instancia, se motivara el comentario personal en el texto.

En los programas de magíster, el progreso en la escritura académica es una necesidad básica como herramienta de comunicación y reflexión crítica. Una ventaja obvia de su uso es que hace posible registrar de forma permanente las intervenciones a las que acceden los investigadores, lo que contrasta con la naturaleza efímera del debate en el aula.

\subsection{LA TECNOLOGÍA COMO ANDAMIAJE EN LA ESCRITURA}

Durante las diferentes etapas del proceso de elaboración del texto, se utilizaron diversas herramientas tecnológicas: primero, en la búsqueda de fuentes especializadas y sitios validados en la red; luego, en el proceso de planificación y organización del ensayo. Más tarde, la mediación tecnológica se produjo durante las etapas de escritura propiamente tal, cuando se estaba generando el escrito en el espacio virtual del blog, en una secuencia que articuló las fases de elaboración con el monitoreo del profesor tutor y de los pares, considerando revisiones, sugerencias y aportes que guiaron las modificaciones, especialmente desde el grupo de aprendizaje, permitiendo así un avance colaborativo del conocimiento (Palamidessi, 2006).

La tecnología fue un factor determinante en la elaboración del esquema digital de escritura $(E D E)$, que es una estructura modelo básica que sirve de andamiaje y orientación al estudiante en su proceso de producción. En la experiencia, el EDE se presentó bajo el formato de una plantilla digital que ofrecía la composición formal del texto que se elaboraría, marcando con determinados colores las dimensiones estructurales de composición del género. El esquema también contenía una serie de iniciadores, conectores y modificadores que proporcionaron apoyo a los sujetos en el manejo del estilo genérico en desarrollo (Wray y Lewis, 2005). Esta plantilla operó dentro de un blog, en el que se organizaron todos los 
contenidos de aprendizaje de escritura. Además, el sitio poseía un espacio de biblioteca, donde se adjuntaron los materiales, un espacio donde estaban las cuentas individuales con las que cada estudiante ingresaba a su EDE e iba activando su proceso de escritura, el que fue retroalimentado semanalmente por el profesor tutor. Esta aplicación poseía también un espacio de foro para los comentarios que iban surgiendo del trabajo colaborativo.

Tanto el blog, como plataforma de interacción, y el EDE, como plantilla de producción textual, son considerados artefactos tecnológicos portadores de una dimensión conceptual y material. Este doble carácter es proporcionado por las funciones con que ambos artefactos fueron concebidos en el diseño: son conceptuales, es decir, ideales en la medida que forman parte de una secuencia de contenidos abstractos, a partir de la cual su forma material ha sido moldeada para cumplir con las interacciones comunicativas a las que servirán de base. De este modo, las citadas herramientas tecnológicas actúan como mediadores de las metas del aprendizaje (Cole, 2000).

\subsection{COMUNIDAD VIRTUAL Y APRENDIZAJE COLABORATIVO}

En el marco de la sociedad del conocimiento, las herramientas de alfabetización académica apoyadas por la tecnología poseen un rol significativo en la creación de nuevos saberes. Se trata de un recurso poderoso en cuanto a su diversificación, ya que contribuye a las distintas áreas del saber humano, lo que resulta preponderante en el desarrollo de nuevos avances científicos. Sin embargo, aun cuando consideramos que estamos frente a una sociedad que se desplaza hacia el conocimiento como principal recurso productivo, cabe resaltar que éste no es consecuencia del trabajo de un solo individuo. Por el contrario, se requiere del apoyo cognitivo de otros para lograr productos significativos. En esta experiencia en particular, esos otros, con quienes se comparten objetivos, intereses y metas comunes, permitieron el nacimiento de una comunidad para el aprendizaje, la cual generó y socializó conocimientos mediante el trabajo en equipo (Gros, 2008).

Recogiendo esta necesidad, el diseño consideró la organización de dicha comunidad de aprendizaje compuesta por todo el grupo curso y conformada en su interior por seis grupos primarios que se constituyeron por afinidad. En el grupo mayor, es decir, en el curso completo, semanalmente se comentaban en términos generales los avances y los aspectos que debían ser mejorados por todos los estudiantes y se avanzaba en la revisión de perspectivas estructurales y formales del texto en desarrollo, ejercicios de modelamiento y tareas de ejercitación. Cada uno de los grupos primarios debía reunirse luego de las clases presenciales y mediar el progreso de los trabajos individuales con el apoyo de sus pares.

Los integrantes del grupo primario se constituyeron en lectores de los textos de sus compañeros, para lo cual usaron las pautas y rúbricas de evaluación con que contó el diseño en la sección de materiales. De esta forma, los estudiantes se apropiaron de los criterios, procedimientos e indicadores con que el texto final sería evaluado y, además, colaboraron con las producciones de sus compañeros, marcando los párrafos que no entendían, formulando preguntas asociadas con el contenido, solicitando aclaraciones respecto de alguna idea que les resultara confusa o incompleta. Por consiguiente, durante todo el proceso de escritura, los sujetos se vieron apoyados por el referente de lectores presenciales de su texto en desarrollo, situación no común, pues significa contar con una ayuda que rompe con la distancia canónica de la escritura en soledad. Desde la dinámica de un taller de escritura, los pares se transformaron en lectores cómplices de los textos en producción. 
Así organizados, el grupo total conformó un espacio de encuentro en la virtualidad, donde los diferentes actores de la experiencia hicieron uso de variados materiales: carpetas con bibliografía, instrucciones de las lecciones, plantilla de escritura, comentarios del foro, etc. Todas estas instancias fueron creadas para albergar y dar forma a la acción comunicativa de carácter sistémica interactiva en función de la meta común de escritura.

En este escenario concebimos el aprendizaje colaborativo virtual como un proceso social de construcción de conocimiento, en el que, a partir del trabajo conjunto y el establecimiento de objetivos comunes, se da una:

reciprocidad entre un conjunto de individuos que saben diferenciar y contrastar sus puntos de vista de tal manera que llegan a generar un proceso de construcción de conocimiento. Un camino en el que cada individuo aprende más de lo que aprendería por sí solo, fruto de la interacción de los integrantes del equipo (Gutiérrez, 2010:114).

En la conformación de un grupo de esta naturaleza, la interacción se convierte en un elemento clave, pues es la herramienta esencial para reunir las contribuciones de los participantes en la co-creación de conocimiento (Gunawardena, 1995).

En una primera instancia, la interacción entre los sujetos se afianza en los aspectos formales del diseño, dependientes del guion metodológico planificado por el docente. No obstante, en un escenario colaborativo virtual, el proceso de escritura está condicionado por aspectos no formales que surgen en la interacción cuando, a partir de su funcionalidad, se genera un lazo afectivo de cohesión en el grupo y los miembros se comprometen con los aprendizajes de sus pares. Entonces, se genera la capacidad de los participantes de proyectarse a sí mismos social y emocionalmente en una comunidad de investigación que crea un clima y una sensación de pertenencia a un grupo que favorece los aprendizajes.

En la dinámica de la interacción, son importantes los códigos experienciales, relacionados con las vivencias y conocimientos previos de los participantes. Así, cada sujeto es portador de códigos culturales representativos de una dimensión histórica que se proyecta en el entramado invisible del colectivo, permitiendo que una comunidad sea lo que es y no otra cosa. Los códigos exigen, por parte del sujeto, un esfuerzo de empatía, lo que significa no aceptar como irrefutable el punto de vista del otro, sino que parte de estos para generar puntos de encuentro, convergencias, comprensión mutua y, sobre todo, para asentar las bases para generar reconceptualizaciones que permitan abrir el conocimiento a nuevas perspectivas (Oliveira, 2009).

\section{ESTRUCTURA DEL DISEÑO: CONTEXTO, ANDAMIAJES Y ARTEFACTOS PARA LA CONSTRUCCIÓN COLABORATIVA}

En la actualidad, en el contexto universitario, la necesidad de progreso en la alfabetización académica, competencia básica para la adquisición de conocimiento profesional, se liga de manera natural a la virtualidad (Figueroa, Aillon y Salazar, 2012). Este componente es el andamiaje base de nuestro diseño, que estructura la colaboración e interacción en línea y permite mantener una tensión dinámica a lo largo de las lecciones de aprendizaje para movilizar la elaboración sistemática de un ensayo, lo cual significa reconocer el potencial de la tecnología e informática y ponerlas en forma organizada y metódica al servicio de 
la producción de un género discursivo. Este medio tecnológico ofrece un espacio para interactuar que facilita la distribución del conocimiento y la experiencia entre los miembros de una comunidad de aprendizaje (Lipponen, 2002).

Las características más definitorias de los nuevos documentos multimedia son su estructura hipermedia y su interactividad, las que determinan una forma de presentar la información diferente a la tradicional. Mientras que los documentos verbal y audiovisualmente codificados hacen una propuesta de recorrido, los documentos multimedia presentan diferentes alternativas de navegación a través de la información, todas ellas igualmente válidas (Gutiérrez, 2010), es decir, presentan la información en estructura de red.

Por consiguiente, crear un diseño didáctico en un contexto de aprendizaje colaborativo virtual significa entender el término contexto no sólo como algo que rodea al fenómeno del aprendizaje, sino también, como lo indica la raíz latina del término, esto es 'entrelazar', significa establecer cómo el todo conectado da coherencia a sus partes (Cole, 2000). El contexto es visto, entonces, como una relación cualitativa entre entidades analíticas, lo que representa en esta experiencia la dimensión retórica del género discursivo (ensayo), mediado por los artefactos tecnológicos usados como apoyos. A su vez, todos estos elementos son puestos en una escena progresiva que sintoniza un aprendizaje colaborativo. Por ende, el acto de escritura, desde su contexto, es entendido en términos de entrelazamiento y requiere una interpretación relacional de la mente. De este modo, la escritura y el contexto se presentan juntos como parte de un único proceso psico-socio-cultural de progreso (Cole, 2000).

\section{DIMENSIÓN PRÁCTICA: IMPLEMENTACIÓN DEL DISEÑO}

\subsection{OBJETIVOS DE LA INVESTIGACIÓN}

- Aplicar un diseño didáctico b-learning con apoyo de una comunidad virtual de aprendizaje para la elaboración de un ensayo en el ámbito de la alfabetización académica hipertextual.

- Describir cómo evolucionan los procesos de aprendizaje colaborativo en propuestas de escritura apoyadas por artefactos tecnológicos.

\subsection{MUESTRA}

La muestra está compuesta por 19 sujetos: profesores de distintas especialidades (Biología, Química, Ed. Diferencial, Ed. Básica, Filosofía, Música, Inglés, Arte, Historia), un ingeniero en alimentos y un fonoaudiólogo. Se trata de un grupo heterogéneo en cuanto a las especialidades, que presenta la necesidad y motivación de avanzar en la escritura académica como requisitos de calificación en el programa.

\subsection{METODOLOGÍA}

Como la dimensión práctica expuesta en el presente artículo describe desde una perspectiva cualitativa las percepciones de los sujetos inmersos en el diseño, en relación con sus logros en la escritura del ensayo, la investigación se adscribe al modelo denominado "investigación de diseño", en el cual las preguntas de investigación refieren a las condiciones que facilitan 
u obstaculizan la concreción de aprendizajes determinados a través de un diseño didáctico. Por tanto, debido a que este tipo de estudio no sólo implica productos, sino también la respuesta humana, las técnicas de investigación son necesariamente diversas (Campbell, 2001).

El diseño en modalidad b-learning consta de 16 sesiones presenciales de dos horas pedagógicas una vez a la semana, seguidas de un número variable de horas de trabajo on line. Durante las clases presenciales, se revisaron aspectos teóricos relacionados con la estructura del ensayo, recursos y modalidades del texto argumentativo, aspectos gramaticales relacionados con conectores y marcadores discursivos, entre otros. Luego, desde la mediación virtual, se ejecutaron los ejercicios de escritura, se monitorearon y retroalimentaron.

En cuanto al texto, los estudiantes seleccionaron el tema del escrito, que definimos como un ensayo académico científico: 'académico', por el contexto especializado del Programa de Magíster en el cual se inserta, y 'científico', ya que tiene como meta la exposición de una reflexión crítica acerca de un problema o necesidad educacional en el ámbito disciplinario de su especialidad pedagógica, reflexión que surge de variadas lecturas especializadas. Una vez elegido el tema, se generó una pregunta de investigación que se debía resolver en la revisión bibliográfica y cuya respuesta se debía plasmar en el escrito.

Para activar el trabajo colaborativo grupal, se conminó a los profesores a que conformaran seis grupos primarios de trabajo, dentro de los cuales el avance en las producciones personales fue periódicamente socializado. De esta forma, los sujetos fueron progresando en las lecciones, en forma individual y grupal, gracias a los andamiajes y artefactos tecnológicos ofrecidos. En el plan de las lecciones, durante las clases presenciales, se proyectó y sistematizó el manejo de las estructuras generales del texto en cuestión, se modelaron y ejercitaron las etapas de la escritura, las que se revisaron y comentaron con todo el curso. Luego, estos ejercicios se replicaron y potenciaron en el grupo primario. Cada nivel o lección requirió del avance de los participantes en el manejo de ciertas habilidades técnicas, tanto de escritura como de los artefactos tecnológicos puestos al servicio de la escritura.

El diseño didáctico hipertextual, desde el inicio, fomentó el acceso no lineal a la información, y privilegió la interconexión y la bi-direccionalidad en las comunicaciones, con el fin de monitorear el proceso de aprendizaje y promover el trabajo en grupo desde la red. En cuanto a la interacción presencial/virtual, ésta se planificó desde dos perspectivas básicas: 1) la interacción colectiva, que condujera a los miembros del curso, en general, y del equipo primario, en particular, a conformar una comunidad que integrara el monitoreo del aprendizaje, y 2) la interacción con los materiales de aprendizaje del diseño desde el contexto del blog.

Durante la aplicación del diseño, a través de la observación participante, se recogieron los datos mediante diarios de campo, el historial del progreso individual de las producciones registrado en el blog, entrevistas semi-estructuradas a los participantes y la revisión de las producciones finales.

\section{RESULTADOS}

De acuerdo a lo descrito en la metodología, el diseño se aplicó a un curso de Magíster en Educación, organizado en seis grupos de trabajo. A pesar de esto, el presente análisis de los resultados sólo considera tres de estos grupos, los más representativos del proceso. 
Los aspectos en torno a los cuales se realiza el análisis corresponden a los núcleos de la investigación que orientaron la elaboración y validación del diseño didáctico, a saber: escritura académica, tecnología y aprendizaje colaborativo.

\subsection{GRUPO 1}

Estaba conformado por una profesora de Educación Básica, un profesor de Artes y una profesora de Educación Diferencial. El grupo logró avances significativos en sus ensayos finales, en los que abordaron temas de sus respectivas especialidades.

a) Escritura académica. Sus percepciones coincidieron con las evaluaciones de proceso y finales en relación con los logros. Éstos marcan una diferencia significativa con las prácticas escritas iniciales, las que registraban niveles descendidos. Valoraron el aprendizaje alcanzado como una habilidad que apoya su desempeño profesional y se mostraron muy satisfechos con el producto final. Se trata del grupo que registra la mayor frecuencia de consultas al profesor tutor durante la primera parte del curso; luego, fueron logrando mayor autonomía e independencia.

b) Tecnología. El profesor de Arte, especialista en diseño gráfico, poseía competencias digitales avanzadas, por lo que apoyó en este aspecto a la profesora de Educación Diferencial, que iniciaba el curso con niveles bajos en esta área. El grupo interactuó con los materiales del blog y los integrantes se organizaron para trabajar por $c h a t^{1}$. Además, para desarrollar un trabajo sistemático respecto de las tareas grupales, los integrantes se reunían semanalmente de forma presencial, fuera de la clase, para comparar sus avances individuales y activar los aportes grupales.

c) Aprendizaje colaborativo. El grupo generó un liderazgo democrático, pues compartían las tareas con un alto grado de compromiso. Sus presentaciones en el grupo ampliado se caracterizaron por un buen nivel de preparación y coordinación que se tradujo en logros cuali y cuantitativos en sus tareas individuales de escritura. Lograron un equilibrio en los intercambios solidarios de apoyo en los procesos lectura y/o escritura. Se trataba de un grupo fuertemente cohesionado, tanto académica como afectivamente.

\subsection{GRUPO 2}

Estaba conformado por dos profesoras de Pedagogía en Química y una de Biología. Lograron muy buenos avances en sus ensayos finales, los que se caracterizaron por un discurso disciplinario con alto grado de especialización en el área de la enseñanza de la química y biología.

a) Escritura académica. Las profesoras reconocieron que el escrito les permitió revisar y ordenar concepciones didácticas que usaban desde la experiencia cotidiana informal, sin

El Chat es definido como "una forma de conversar en tiempo real en la red. Se trata de algo completamente diferente a un correo, ya que permite mantener un diálogo inmediato e interactivo con un grupo de personas distribuidas por cualquier lugar del mundo" (Menning, 2000: 47). 
haberlas traducido a reflexiones más organizadas y sistemáticas. Declararon que la escritura académica les permitió, por un lado, profundizar conceptos que desde la práctica del aula abordaban de manera parcial, para visualizarlos así desde perspectivas enriquecidas en el diálogo con la literatura especializada; y, por otro lado, tomaron conciencia de la dimensión epistémica de la escritura al replantearse, desde el texto, con una mirada crítica hacia su práctica pedagógica.

b) Tecnología. El grupo estuvo obligado a usar la tecnología para interactuar, ya que las tres integrantes eran de diferentes ciudades, sin posibilidades de interacción presencial fuera de las sesiones de clases. Cada una poseía niveles de alfabetización digital suficientes para el manejo de la plataforma, se comunicaban e interactuaban con frecuencia a través del foro del blog, por lo que el tutor tuvo acceso en detalle a las necesidades, dificultades y avances que mediaron el proceso. Ellas también se comunicaron vía Messenger y correos electrónicos.

c) Aprendizaje colaborativo. Se produjo un liderazgo democrático compartido. La formación científica común les proporcionó una base en los niveles de alfabetización académica de su disciplina, lo que se tradujo en mejores niveles de inicio en cuanto a la definición del tema y la pregunta de investigación que guió la búsqueda bibliográfica y, luego, en la dimensión de comprensión, un buen dominio del nivel léxico-semántico de los textos consultados. Sus ensayos alcanzaron un desempeño destacado. Las integrantes lograron colaborar entre ellas con propiedad en las producciones individuales, desde el dominio disciplinario común. En el nivel discursivo, compartieron sus hallazgos y fueron rigurosamente críticas con los textos del grupo.

\subsection{GRUPO 3}

Estuvo integrado por un profesor de Ciencias, un profesor de Música y dos profesoras de Educación Básica. Fue el grupo en el que se dieron los más bajos rendimientos. Una de las profesoras de Educación Básica abandonó el curso sin terminarlo, mientras que la otra docente aprobó con la nota mínima. El profesor de Música reprobó.

Se trataba de un grupo muy heterogéneo. El profesor de Ciencias, desde un comienzo, se distinguió por su buen desarrollo de la escritura y avanzó separado del grupo sin apoyar al resto. Los otros tres profesores, con competencias muy descendidas, no lograron afianzarse como equipo.

a) Escritura académica. Poseían percepciones variadas y disímiles respecto de la escritura académica. El profesor de Ciencias valoraba la herramienta y buscó su avance individual, no presentó interés en apoyar a los otros integrantes. Los tres profesores restantes también declararon valorar la herramienta, pero, en la práctica, no asociaron el progreso de la escritura al trabajo constante y sistemático. El curso no logró desarrollar en ellos la disciplina suficiente para enfrentar de manera sistemática las tareas de escritura.

b) Tecnología. No usaron todas las herramientas tecnológicas que el diseño puso a su disposición. Utilizaron el blog para la mantener el contacto con el profesor tutor y para recibir las retroalimentaciones de sus tareas y ejercicios, que generalmente realizaron atrasados con diferentes excusas, por lo que se les otorgó plazos extras. El EDE les ocasionó problemas, 
por consiguiente, no siguieron las instrucciones y se les debió tolerar que escribieran de manera libre, sin ceñirse a la plantilla.

c) Aprendizaje colaborativo. No hubo liderazgo. El grupo no logró constituirse como tal, sólo presentaron intereses individuales. No asignaron importancia a los apoyos tecnológicos, como el foro, para conectarse entre ellos, tampoco utilizaron otros canales asumidos por los grupos restantes. Las tareas y revisiones colectivas fueron producto de improvisaciones de última hora.

En síntesis, de los seis grupos en que se organizó el curso, cinco de ellos concluyeron la producción del texto planificado dentro de los niveles de aprobación establecidos. Las descripciones de los grupos 1 y 2 presentados anteriormente ejemplifican este desempeño. Lo contrario ocurre con el grupo 3, en el que estas condiciones no se lograron. Interesa a continuación analizar algunas de las variables que, a nuestro juicio, inciden en estos resultados.

\section{DISCUSIÓN}

\subsection{LA ESCRITURA ACADÉMICA Y EL DISEÑO DIDÁCTICO HIPERTEXTUAL}

Retomamos la premisa del epígrafe, esto es, que lo que se aprende es inseparable del modo en que se aprende (Marton \& Booth, 1997), para reafirmar la importancia de la metodología en una experiencia de esta naturaleza. En un diseño b-learning, es prioritario establecer la coherencia entre los objetivos del curso, los materiales, las tareas de los estudiantes y la acción docente. Es decir, se necesita configurar un ambiente de aprendizaje funcional tanto en su entorno presencial como virtual, de manera que se favorezcan las relaciones de los sujetos intervinientes.

Puesto que el diseño surge de la necesidad de desarrollo de la escritura académica en un contexto universitario, éste se encuentra condicionado por la disciplina y su didáctica. De esta forma, la tecnología está ligada a la pragmática del lenguaje que desde la gestión docente debe considerar a los agentes, así como los factores, psicológicos y socioculturales que determinan los pasos y normas del trabajo individual y colectivo en la escritura formal de un ensayo.

La lectura y la escritura académica en la secuencia didáctica progresan desde la búsqueda de información, a la producción de un texto en un género que incluye el manejo de las modalidades discursivas expositiva y argumentativa. Esta competencia implica el dominio de un saber y un saber hacer, que moviliza en el sujeto procesos metacognitivos, capacitándolo para resolver problemas concretos de diferentes grados de complejidad con una actitud favorable y proactiva hacia el manejo de la herramienta escrita (Cabello, 2012). Sin embargo, como sostiene Cassany,

leer y escribir en la red es más difícil que hacerlo con papel y bolígrafo (...). En la red navegamos a través de millones de fuentes de información, usando herramientas sofisticadas que no siempre dominamos, como los motores de búsqueda, los traductores asistidos por los mapas de web (...). Se homogenizan los artefactos letrados y los contextos de lectura 
convergen en la pantalla: la lectura y escritura pierden más referentes situacionales y exigen más contextualización cognitiva; se incrementa la dificultad de comprender, situar e interpretar los textos (2012: 225).

Todas estas cuestiones son necesarias de tener en cuenta cuando usamos la tecnología en las prácticas letradas.

El blog que se implementó como plataforma del curso contenía una estructura simple de tres interfaces: biblioteca, foro y el espacio de escritura para cada integrante del grupo. En este último espacio virtual, se encuentran los EDE para elaborar comentarios y el ensayo final, facilitando la interacción multidireccional y flexibilizando la dimensión espaciotemporal de participación.

En nuestra muestra, el EDE funcionó de manera eficiente para cinco de los seis grupos. La plantilla con sus iniciadores, conectores y modificadores (elementos gramaticales), marcados en la diagramación con diferentes colores, proporcionaron la estructura textual del ensayo. De esta forma, los citados recursos cumplieron el papel de un simulador de elaboración al re-presentar una huella discursivo formal, que se constituyó en una especie de contenedor del pensamiento reflexivo. Con esta herramienta, se proporcionó al estudiante un soporte efectivo en el desafío de lanzarse a la 'página en blanco' para expresar reflexiones críticas producto de lecturas académicas.

$\mathrm{Al}$ respecto, destacamos que en los ensayos finales las ideas expuestas por los sujetos cumplieron con los objetivos de autentificar un conocimiento existente respecto del tema en cuestión y, al mismo tiempo, de generar un nuevo conocimiento en una base de escritura asincrónica.

\subsection{EL DISEÑO TECNOLÓGICO Y EL APRENDIZAJE COLABORATIVO}

La aplicación del diseño permitió reconocer los aspectos esenciales que tienen que ver con el aprendizaje colaborativo, en tanto

conjunto de métodos de instrucción o entrenamiento para uso en grupos, así como de estrategias propicias para el desarrollo de habilidades mixtas (aprendizaje, desarrollo personal y social); donde cada miembro del grupo es responsable de su propio aprendizaje, así como el de los restantes miembros del grupo (Johnson, Johnson y Holubec, 1999: 31).

En la secuencia didáctica, destacamos dos aspectos básicos que condicionan los procesos y sus resultados. Por un lado, es significativo el diseño en cuestión, que contextualiza y organiza las tareas que derivarán en los aprendizajes esperados. Además, es relevante y fundamental el grado de compromiso personal y colectivo que los sujetos adquieren en relación con su aprendizaje y con los de sus pares, compromisos que se evidencian en actitudes de motivación hacia el trabajo colectivo.

El diseño probó ser efectivo en la conformación de comunidades de aprendizaje. Proporcionó los recursos y herramientas que permitieron al estudiante aprender de manera individualizada y, al mismo tiempo, brindó la oportunidad de discutir y compartir al interior del grupo, construir y progresar colaborativamente, controlando su propio proceso de aprendizaje y, a la vez, cumpliendo un rol activo en la elaboración de los textos de sus pares. El grado de compromiso que los estudiantes lograron dentro de su grupo primario 
y con el grupo mayor condicionó su motivación con los objetivos comunes, potenció su autonomía, control y reflexión sobre sus procesos y los del grupo.

La experiencia de construir secuencialmente un ensayo desde una perspectiva personal y, paralelamente, ir afinando y contrastando esa construcción con la interacción en una comunidad, tiene que ver con el denominado "constructivismo cooperativo o transaccional". Éste plantea el reconocimiento de la estrecha relación que existe entre la construcción personal del significado y la influencia que ejerce lo social en la configuración de la relación educativa (Garrison y Anderson, 2005).

El trabajo colaborativo implicó desarrollar el interés por los problemas formales y semánticos que iban surgiendo en los escritos de los diferentes miembros del grupo. La conformación multidisciplinaria de los sujetos enriqueció los productos con aportes desde perspectivas diferentes y/o complementarias.

Constatamos, además, que la escritura académica mediada por el aprendizaje colaborativo con apoyo tecnológico, desde su función epistémica, activa dos principios: por un lado, el de la interacción, el cual unifica en un marco temporal inmediato los mundos personal (subjetivo) y social (objetivo), es decir, mediante esta interacción, el conocimiento discursivo es construido y confirmado. Por otro lado, se activa el principio de continuidad, por cuanto el resultado es la capacidad que permite al sujeto seguir formándose en la escritura, como base de experiencias profesionales nuevas y valiosas. Ambos principios se dan en la escritura académica tradicional, pero en plazos y espacios mucho más extensos.

De esta forma, la tecnología posibilita entornos virtuales de aprendizaje, en los cuales la interacción entre las personas y de éstas con los materiales se realiza de una manera más ágil, optimizando los procesos de relación entre objeto y sujeto de formación, con mejores logros en el escrito final.

\subsection{ALCANCES Y LIMITACIONES DEL MODELO}

A modo de colofón y sin que éste quiera constituirse en una excusa, lo ocurrido en el grupo 3 sin duda amerita reflexiones específicas. A nuestro juicio, falló la gestión del tutor, en otras palabras, se pudo haber tomado medidas tempranas de redistribución del grupo o medidas para potenciar el liderazgo al interior de éste, de forma tal que los integrantes encontraran mayores puntos de convergencia y descubrieran cómo aprovechar su diversidad. Esta situación exige de un mayor conocimiento por parte del docente de la dimensión histórica cultural de los sujetos del grupo, de sus experiencias y limitaciones previas. No se debió suponer ni esperar un despegue de iniciativa que en el grupo finalmente no se produjo.

Para potenciar la construcción pedagógica del conocimiento, los profesores hablamos de crear 'diseños de aprendizaje', es decir, crear espacios con intencionalidad educativa. Podemos diseñar situaciones, pero no podemos asegurar los efectos que van a producir. El éxito o fracaso de las actividades en un proceso de enseñanza-aprendizaje estará determinado por un amplio conjunto de variables, cuyo grado de interrelación posee un cierto nivel de indeterminación, que debe tomarse en cuenta en el propio diseño, ya que la incertidumbre y la indeterminación forman parte del propio sistema. Por consiguiente, como señala Wenger, "existe una incertidumbre intrínseca entre el diseño y su realización en la práctica, porque la práctica no sólo es el resultado del diseño, sino una respuesta al mismo" (2001: 43). 
En el diseño colaborativo virtual, el escenario es más complejo que los escenarios de aprendizaje tradicionales, son muchas más las variables que se conjugan y, por tanto, más los riesgos presentes cuando se interactúa con personas. Retomando a McLuhan y Powers, al trabajar con los medios existen elementos que se potencian, dejando atrás otros y, al mismo tiempo, algo de lo viejo se trae al presente. Los autores sostienen que:

cualquier medio: a) intensifica algo en una cultura mientras que al mismo tiempo b) vuelve obsoleta otra; c) recupera una fase o factor dejado de lado desde tiempo atrás y d) sufre modificaciones (o inversiones) cuando se le lleva más allá de los límites de su potencial (1990: 67).

¿Qué se trae al presente? Sin duda elementos de la tradición oral que yacen empolvados en la masiva aula académica de hoy. La empatía, la interacción afectiva que redunda en el compromiso y motivación social frente a metas de aprendizaje comunes; aspectos que a menudo subyacen escondidos por el culto al individualismo de la fragmentada sociedad contemporánea.

En la experiencia que nos ocupa, sin duda la flexibilidad y rapidez de la interacción de los agentes del aprendizaje entre sí y con los materiales son condicionantes que la dimensión tecnológica potencia. A su vez, posibilita la conformación de la comunidad de aprendizaje sin limitaciones espacio temporales, lo que en el ámbito de la escritura académica implica dejar atrás la escritura en soledad, al proporcionar lectores durante el proceso de producción.

Con más espacio y flexibilidad para la comunicación en un tiempo 'sin tiempo', los actores de la comunidades virtuales necesariamente deben premunirse del principio de alteridad. Esto implica ir al encuentro con los otros, re-conocerse en los otros, principio indispensable para constituirnos como sujetos a través del diálogo científico.

En síntesis, un diseño de esta naturaleza cumple con la necesidad de incorporar diversos códigos representacionales a partir de las interacciones con los símbolos, las herramientas de la cultura y los otros sujetos, en aras de la riqueza de la complejidad que favorece la denominada cognición distribuida (Bruner, 1997). Así también favorece el carácter contextualizado del aprendizaje, a partir del cual la situacionalidad del conocimiento no se entiende limitada a contextos inmediatos, sino en la proyección del conocimiento declarativo y procedimental en contextos de usos reales en diferentes disciplinas, aspecto que se denomina cognición situada (Bruner, 1997).

\section{REFERENCIAS BIBLIOGRÁFICAS}

Bereiter, C. (2002). Education and Mind in Knowledge Age. Hillsdale, N. J.: Lawrence Erlbaum Associates.

Bruner, J. (1997). La educación puerta de la cultura. Madrid: Visor.

Cabello, R. (2012). Palos en la rueda. Cinco factores de resistencia a la Internet en la escuela. En D. Goldin, M. Kriscautzy y F. Perelman (Comps.), Las TIC en la escuela, nuevas herramientas para viejos y nuevos problemas (pp. 183 -216). Barcelona: Océano Travesía.

Campbell, D. S. (2001). Diseños experimentales y cuasi-experimentales en la investigación social. Buenos Aires: Amorrortu.

Cassany, D. (2012). La metamorfosis digital: Cambios, ventajas y riesgos de leer y escribir en la red. En D. Goldin, M. Kriscautzy y F. Perelman (Comps.), Las TIC en la escuela, nuevas 
herramientas para viejos y nuevos problemas (pp. 217-236). Barcelona: Océano Travesía.

Cole, M. (2000). Psicología cultural. Madrid: Morata.

Figueroa, B., Aillon, M. y Salazar, O. (2012). Avances hacia la comprensión del fenómeno de la alfabetización académica hipertextual en el contexto de formación docente. Universum. Revista de Humanidades y Ciencias Sociales, vol.1 (27), 55-70.

Garrison, D. y Anderson, T. (2005). El E-learning en el siglo XXI: Investigación y práctica. Barcelona: Octaedro.

Gros, B. (2008). Aprendizajes, conexiones y artefactos. Barcelona: Gedisa.

Gunawardena, C. N. (1995). Social Presence Theory and Implications. Internacional Journal of Educational Telecommunications, vol.1 (2/3), 147-166.

Gutiérrez, A. (2010). Creación multimedia y alfabetización en la era digital. En R. Aparici (Coord.), Educación más allá del 2.0 (pp.171-186). Barcelona: Gedisa.

Johnson, D. W., Johnson, R. T. y Holubec, E. J. (1999). El aprendizaje cooperativo en el aula. Barcelona: Paidós.

Lipponen, L. (2002). Exploring Foundations for Computer Supported Collaborative Learning. In G. Stahl (Ed.), Computer Support for Collaborative Learning: Foundations for a CSCL Community. Proceedings of CSCL (pp. 72-81). Hillsdale N. J.: Lawrence Erlbaum Associates.

Marton, F. \& Booth, S. (1997). Learning and Awareness. Maywah, N. J.: Lawrence Erlbaum Associates.

McLuhan, M. y Powers, B. R. (1990). La aldea global. Barcelona: Gedisa.

Menning, V. M. (2002). Diccionario de Internet e Intranet. Barcelona: Salvat Editores.

Oliveira, I. (2009). Caminos de la educomunicación: Utopías, confrontaciones, reconocimientos. Revista Nómadas, (30), 194- 207.

Palamidessi, M. (2006). La escuela en la sociedad de las redes. Una introducción a las tecnologías de la información y comunicación en la escuela. Buenos Aires: Fondo de Cultura Económica. Paidós.

Wenger, E. (2001). Comunidades de práctica. Aprendizaje, significado e identidad. Barcelona:

Wray, D. y Lewis, M. (2005). Aprender a leer y escribir textos de información. Madrid: Morata. 
